\title{
Neuronal chemotaxis by optically manipulated liposomes
}

\section{G. Pinato}

L. T. Lien

E. D'Este

V. Torre

D. Cojoc

cojoc@tasc.infm.it
CNR, Istituto Officina dei Materiali (IOM), Laboratorio TASC, Area Science Park- Basovizza, S.S. 14, Km 163.5, 34149, Trieste, Italy

International School for Advanced Studies (ISAS-SISSA), Neurobiology Sector, Via Bonomea 265, 34136, Trieste, Italy

CNR, Istituto Officina dei Materiali (IOM), Laboratorio TASC, Area Science Park- Basovizza, S.S. 14, Km 163.5, 34149, Trieste, Italy

International School for Advanced Studies (ISAS-SISSA), Neurobiology Sector, Via Bonomea 265, 34136, Trieste, Italy

CNR, Istituto Officina dei Materiali (IOM), Laboratorio TASC Ed. MM, Area Science Park- Basovizza, S.S. $14, \mathrm{Km} 163.5,34149$, Trieste, Italy

We probe chemotaxis of single neurons, induced by signalling molecules which were optically delivered from liposomes in the neighbourhood of the cells. We implemented an optical tweezers setup combined with a micro-dissection system on an inverted microscope platform. Molecules of Netrin-1 protein were encapsulated into micron-sized liposomes and manipulated to micrometric distances from a specific growth cone of a hippocampal neuron by the IR optical tweezers. The molecules were then released by breaking the liposomes with UV laser pulses. Chemotaxis induced by the delivered molecules was confirmed by the migration of the growth cone toward the liposome position. Since the delivery can be manipulated with high temporal and spatial resolution and the number of molecules released can be controlled quite precisely by tuning the liposome size and the solution concentration, this technique opens new opportunities to investigate the effect of physiological active compounds as Netrin-1 to neuronal signalling and guidance, which represents an important issue in neurobiology. [DOI: http://dx.doi.org/10.2971/jeos.2011.11042]

Keywords: Neuronal guidance, drug delivery, optical tweezers, laser micro-dissection

\section{INTRODUCTION}

Changes of the cellular environment, in neurobiology experiments, are usually achieved through bath administration of molecules. All the cells of the culture plate are therefore stimulated in the same manner. This method is well suited for studies on cell population but much less effective for single cell studies. Moreover, complex cells like neurons are characterized by specialized compartments with different receptors which may respond in different ways to the same stimulus [1]. Localized chemical delivery include ejection from micropipettes and puffers [2] and, more recently, microfluidic devices $[3,4]$.

Spatially restricted and fast stimulation can be obtained by photoactivation of caged compounds [5], which can be both extracellular and intracellular. The temporal resolution of delivery is very high ( $\mu \mathrm{s}$ to $\mathrm{ms}$ ) and the release location is restricted to the area of incidence of the uncaging light [6]. However, most of the caged compounds turned out to produce undesired side effects, being able to block both glycine and GABA receptors [7]. Moreover, most neuroactive molecules are not available in caged form, thus precluding a large number of applications.

The use of microvectors for the delivery of a large variety of molecules that can be positioned with submicrometric spatial resolution in contact or near a cell could be a convenient solution for many single cell experiments. Such vectors, e.g. surface functionalized beads, biodegradable particles, or filled liposomes, can be easily manipulated by optical tweezers integrated on the same optical microscopy platform used for imaging.

Silica and latex microbeads are mostly used to demonstrate the capabilities of optical tweezers for single and multiple particle manipulation [8]. Moreover, such microbeads, coated with carboxylic groups, are commercially available [9] and can be cross-linked with a variety of proteins [10]. A distinct feature of functionalized beads, unlike systems that deliver free molecules, is that the ligands can not be endocytosed by the cell. In this way, the pathways activated by the endosomes are excluded and the local effect of the molecules can be specifically analyzed. For instance, large beads $(10 \mu \mathrm{m})$ crosslinked with a neurotrophic protein and manipulated with micropipettes have been used to potentiate the nearby synapses in Xenopus nerve-muscle cultures [11]. Micropipette manipulation, however, provides only minimal flexibility and low spatial resolution. Using optical tweezers, manipulation of smaller microbeads (down to $200 \mathrm{~nm}$ ) could be achieved with 
a higher spatial resolution, as it is allowed by the high NA lenses used in this case.

Apart from coated microbeads, which preserve the active components on their surface, another class of vectors is represented by microsources. The active molecules are encapsulated, in this case, in a dense polymeric network and released afterwards by the lattice biodegradation. Optically manipulated microsources can be used to generate spatial and time adaptable molecular gradients. An interesting application was reported recently to control cell polarization and migration [12]. Microsources releasing the chemoattractant formyl-methionine-leucine-phenylalanine and microsources releasing the actin polymerization inhibitor cytochalasin D were fabricated from polylactic-co-glycolic acid (PLGA) and positioned close to a human neutrophil cell by using single and multiple optical tweezers. PLGA microparticles are biodegradable sources designed to continuously release molecules over long periods of time (minutes) to create controlled chemical gradients.

Chemical stimuli, encapsulated in optically manipulated liposomes and delivered by breaking the liposome with a pulsed UV laser were proposed as local delivery with precise temporal switch-on control [13]. A submicrometric diameter liposome $(0.6 \mu \mathrm{m})$ was loaded with a Carbachol solution $(0.1 \mathrm{M})$ and positioned at $0.5 \mu \mathrm{m}$ from a CHO-M1 cell, stained with the fluorescent calcium indicator Fluo-3. The content was then delivered by liposome photolysis with an UV laser pulse and the increase of the intracellular calcium level monitored by fluorescence. This method has the advantage that any membrane impermeable molecules, including peptides and proteins, can be easily encapsulated into liposomes and delivered to cells. Moreover, the amount of released molecules can be varied easily by controlling either the size of the liposome or the concentration of the encapsulated compound [14]. Therefore, we have recently begun to extend this technique to investigate cultured neurons which are morphologically complex cells, synaptically connected into a network. For instance, we are interested in studying the neuronal electrical activity induced by small amounts of $\mathrm{KCl}$ molecules released from liposomes, optically manipulated nearby the cells. If the amount of molecules is large enough to produce stimulation, this activity can be imaged by fluorescence microscopy of the cells stained with a Calcium dye. The characterization of such a neuronal electrical activity by local stimulation could find many applications in electrophysiological characterization of neuronal conductances (ion channels and receptors). However, since the effect of the released component is monitored by means of cell staining and consequent Calcium fluorescence imaging, special care should be given to the quantitative interpretation of the data.

Another example, in neurobiology, is the study of the axonal guidance. The control of the axonal growth, characterized by continuous development and retraction of the growth cone and its lamellipodia and filopodia [15], is fundamental to understand the nervous system development and may suggest new strategies to promote axon regeneration after injury or disease. Among other bio-chemical clues responsible for the axonal guidance, Netrin- 1 is an important chemo-attractant molecule, belonging to the Netrins family. Netrins are known as laminin-related axonal secreted proteins, which are critical in controlling axon elongation and pathfinding [16]. Therefore, in this paper, we have chosen to encapsulate this protein in micrometric sized liposomes and deliver it at defined positions in the nearby of the growing axons. Netrin- 1 is a protein of about $80 \mathrm{kDa}$ with versatile chemotaxic effects demonstrated in vitro on hippocampal neurons [17]. The effects of Netrin-1, could hence be observed as morphological changes, monitored by time lapse microscopy.

We have built an optical manipulation setup combining an optical tweezers with a micro-dissection system on an inverted microscope platform to manipulate the liposomes and release their content close to single cells. Setup details and working principle are given in the next sections. Liposomes and cells preparation are described in Section 3. Experimental results, demonstrating that neuronal chemotaxis can be induced by the Netrin-1 molecules released from a small liposome with a diameter of several micrometers, are presented in Section 4.

\section{OPTICAL SETUP}

The optical setup is composed by three main modules (Figure 1):

- custom built IR optical tweezers

- commercial UV micro-dissection system (MMI-CellCut Plus, MMI, Zurich, Switzerland)

- commercial inverted microscope Nikon Eclipse TE-2000E.

The trapping laser beam is generated by a $1024 \mathrm{~nm}$ continuous wave single mode $\mathrm{Yb}$ fiber laser (YLM-5, IPG Photonics $\mathrm{GmbH}$, Burbach, Germany). The laser head has a built-in collimator providing a TEM00 collimated beam with the diameter $\mathrm{D}_{b}=5 \mathrm{~mm}$. Since the entrance pupil of the microscope lens (Olympus, 100X, NA 1, WD 1) has a diameter Dp $=3.6 \mathrm{~mm}$, the laser beam overfills it, meeting the condition for an optimum trapping [18]. The objective lens has a moderate numerical aperture (NA $=1$, water immersion) which still provides for a stable three-dimensional (3D) trapping. In turn, it has a relative long working distance (WD $=1 \mathrm{~mm}$ ), allowing to move the trapped particle axially for hundreds of microns inside the sample cell. As it will be shown in Section 4, this is of advantage for liposome selection at the top and its transportation down to the bottom of the sample cell. The direction of the trapping IR beam is aligned with the direction of the micro-dissection UV beam by two mirrors (M1 and M2 in Figure 1). A convergent lens, L, with the focal length, $\mathrm{f}_{L}=150 \mathrm{~mm}$ is placed just before the entrance aperture of the micro-dissection system to adjust the convergence of the trapping IR beam to the convergence of the UV micro-dissection beam. The alignment of the IR trapping beam is facilitated by a red guide laser beam $(660 \mathrm{~nm}, 0.5 \mathrm{~mW})$, emitted by the same source. Powers between 10 to $20 \mathrm{~mW}$ are necessary at the sample location to stably trap the liposomes. Typically, the IR laser power at the output of the collimation head is regulated from 150 to $300 \mathrm{~mW}$. The micro-dissection system is equipped with 


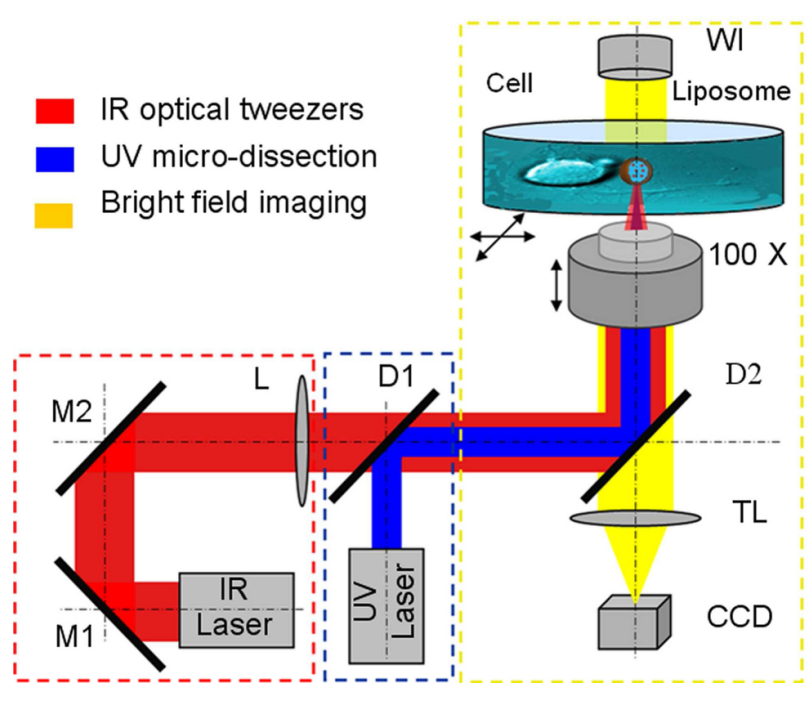

FIG. 1 Optical manipulation setup: the IR laser trapping beam direction is adjusted by two mirrors, $M_{1}$ and $M_{2}$, and its convergence by the convergent lens, L, to be aligned with the UV laser micro-dissection beam. Both laser beams are then directed into the entrance pupil of the microscope objective (100 X) by the dichroic mirrors D1 and D2. The sample is illuminated by a white light source (WI) and the image is formed through the tube lens ( $\mathrm{TL})$ on the $\mathrm{CCD}$ camera.

a solid-state pulsed UV laser (355 nm, max $1 \mu \mathrm{J} /$ pulse, pulse duration $<0.5 \mathrm{~ns}$, repetition rate $<5 \mathrm{kHz}$ ). The beam is collimated by internal optics and directed toward the microscope objective by two dichroic mirrors (D1, D2). The number of required pulses may vary with liposome morphology (single or multi-lamellar). Short series of less than 50 pulses are usually enough to break the liposomes wall and deliver their content. The power and the focus of the UV laser beam are controlled by the MMI software, which controls also the XY motorized sample stage and the image recording on the CCD camera. The sample cell on which the neuronal cell are cultured is a glass-bottom Petri-dish. Since the experiments last for tens of minutes, the Petri-dish is settled on a heating insert and kept at $37^{\circ} \mathrm{C}$ by a digital temperature controller (PeCon $\mathrm{GmbH}$, Erback, Germany) to maintain physiological conditions.

Even if single optical traps are created in this experiment, the setup can be easily adapted to trap and manipulate simultaneously more than one liposome. This is allowed by the high optical power of the IR laser beam (up to $5 \mathrm{~W}$ ) which can be split by a diffractive optical element (DOE). In addition, the beam is linearly polarized and thus does not require an additional component to control the polarization, and a proper designed phase DOEs can be then projected on a phase programmable modulator (PPM) to shape the trapping beam into multiple traps [19, 20]. Beside the PPM, an additional beam expander (3X) and a Fourier transform lens (plan convex lens with focal length $\mathrm{f}=250 \mathrm{~mm}$ ) are the only components to be inserted between laser head and the first mirror, M1, in order to generate multiple optical traps simultaneously.

Note that this setup is characterized by a simple manipulation requiring only a short training, even if the operator does not have an expertise in optics, as it is the case of most biological laboratories.

\section{LIPOSOME AND CELL CULTURE PREPARATION}

Liposomes were obtained from the following lipid mixture: Cholesterol: $9 \mu \mathrm{mol}, \mathrm{L}-\alpha$-Phosphatidylcholine: $63 \mu \mathrm{mol}$, Stearylamine: $18 \mu \mathrm{mol}$ (Sigma-Aldrich). The lipid solution was prepared at the concentration of $10 \mathrm{mg} / \mathrm{mL}$ in chloroform : methanol $(2: 1, \mathrm{v} / \mathrm{v})$. The solution obtained was then saturated with nitrogen and it could be stored at $-20^{\circ} \mathrm{C}$. Liposomes with diameters of $1-10 \mu \mathrm{m}$ were obtained using the lipid film hydration method [21]. Briefly, aliquots of the lipid solution were dried in vacuum for 24 hours in glass vials. The lipid films obtained were finally hydrated with a solution containing the guidance molecule Netrin-1 $(5 \mu \mathrm{g} / \mathrm{mL})$. Sucrose $100 \mathrm{mM}$ was also included in the hydration solution to allow better liposome washing and to improve trapping of large liposomes as previously reported [22]. After brief incubation liposomes were formed. Liposomes were then gently centrifuged $(5000 \mathrm{rpm} \times 3 \mathrm{~min})$ and rinsed 3 times with PBS to wash the external solution. 1-2 uL of the final liposome solution were then administred to the cell cultures. Single liposomes were subsequently identified, trapped and positioned at the location of interest.

Hippocampal neurons from Wistar rats (P0-P2) were prepared [23], in accordance with the regulation of the Italian animal Welfare Act, and under the approval of local veterinary service. Cells were plated at the concentration of $10^{\wedge} 5$ cells $/ \mathrm{mL}$ on glass bottom Petri-dishes, polyornithine/Matrigel precoated. Neuronal cultures were maintained in an incubator at $5 \% \mathrm{CO} 2,95 \%$ moisture and $37^{\circ} \mathrm{C}$ in minimum essential medium with Earle's salts and Glutamax I (MEM, Life Technologies, Invitrogen) with 10\% FBS (Gibco), $35 \mathrm{mM}$ D-glucose (Lancaster), $14 \mathrm{mM}$ Hepes (Sigma), $1 \mathrm{mM}$ vitamin B12 (Sigma), $0.36 \mathrm{mM}$ d-Biotin (Sigma), $30 \mu \mathrm{g} / \mathrm{mL}$ insulin (Sigma), $100 \mu \mathrm{g} / \mathrm{mL}$ bovine transferrin (Sigma), and antibiotics. Cultures were used after 18-24 hours in vitro. Before starting the experiment cultures were bathed in Ringer's solution $(145 \mathrm{mM} \mathrm{NaCl}, 3 \mathrm{mM} \mathrm{KCl}, 1.5 \mathrm{mM} \mathrm{CaCl} 2,1 \mathrm{mM}$ $\mathrm{MgCl} 2,5 \mathrm{mM}$ Glucose, $10 \mathrm{mM}$ HEPES, adjusted to $\mathrm{pH} 7.4$ with $\mathrm{NaOH})$.

\section{NEURONAL CHEMOTAXIS - EXPERIMENTAL RESULTS}

Using the procedure described in Section 3 we synthesized control liposomes (i.e. without Netrin-1), with diameters ranging from 1 to $10 \mu \mathrm{m}$. Few liposomes were introduced at the top of the Petri-dish by gently pushing them from a capillary tip (20 $\mu \mathrm{m}$ inner diameter). Naturally, the liposomes (with a diffusion coefficient $\mathrm{D} \sim 0.1 \mu \mathrm{m}^{2} / \mathrm{s}$ ) remained near the upper surface for long time before spreading into the whole volume of the Petri-dish. A single liposome could thus be visually selected, trapped and manipulated as schematically shown in Figure 2(a). The liposome was kept fixed far from the bottom of the sample cell while this was moved for millimeters in the XY plane, until the region of interest was reached. The liposome was then laid on the bottom of the sample cell by moving axially the microscope lens for hundreds of microns. A sequence of images showing a trapped liposome and how 


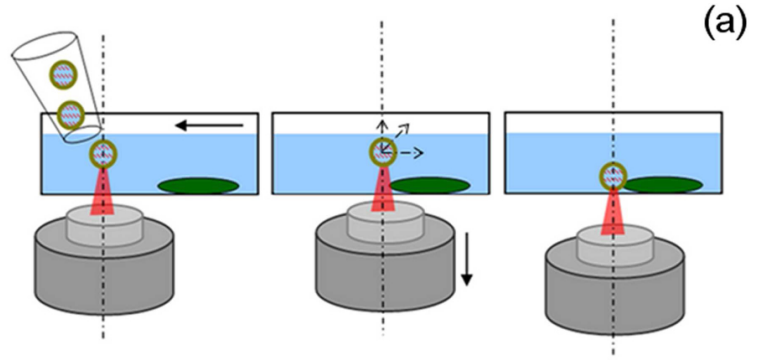

(b)

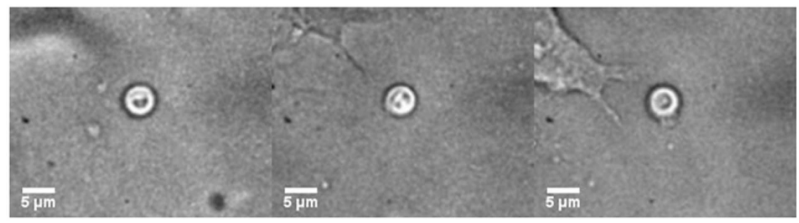

FIG. 2 Optical trapping and X-Y-Z manipulation of the liposme.

(a) Schematic of liposome insertion and optical trapping (frame on the left). Liposome $X-Y$ positioning (frame in the middle) is obtained by moving the sample cell, as indicated by the horizontal arrow. Vertical Z positioning of the liposome to the desired position close to the cell is obtained by moving the objective, as indicated by the vertical arrow.

(b) Sequence of images showing a liposome trapped far away from the bottom of the sample cell and then moved down to the desired position in the vicinity of the cell. Full sequence of the manipulation steps is shown in Movie-1 (500 KB).

its relative position to the sample cell was changed, is shown in Figure 2(b). The full sequence of the manipulation steps is shown also in Movie-1 (500 KB), supplementary information.

Once the liposome was positioned into the desired site, we broke its membrane by a short series of UV laser pulses to release its content around. To rule out any influence of the control liposome or of the laser beam on the neuronal guidance, we monitored the growth cone for 5 minutes after liposome photolysis, taking images every 30 seconds. We repeated ten times the same type of experiment, confirming the absence of any morphological effect induced by the photolysis of empty liposomes on the neuronal growth. An example is shown in Movie-control-1 (222 KB) of the supplementary information.

In the next step we used liposomes filled with Netrin-1 molecules in solution (concentration $5 \mu \mathrm{g} / \mathrm{mL}$ ). To preclude potential leakage/diffusion of Netrin-1 molecules out of the liposome, with the subsequent effect on the neuron morphology, we performed a second type of control experiments. The liposome filled with Netrin-1 was positioned and held near the growth cone for more than 5 minutes before the liposome photolysis. Examining visually the neuron, we controlled that no significant changes of the neuron morphology occurred. An example of such a control experiment is shown in Moviecontrol-2 $(328 \mathrm{~KB})$ of in the supplementary information.

Afterwards, we studied the guidance effect of the Netrin-1 molecules released from the liposomes. The liposome was positioned near a neurite, as shown in Figure 3, and its content released by laser photolysis. To see the effect induced by the Netrin-1 molecules, the neurite growth cone was monitored for 5 minutes after the release, recording images every $30 \mathrm{sec}-$ onds. The time lapse imaging of the experiment is presented

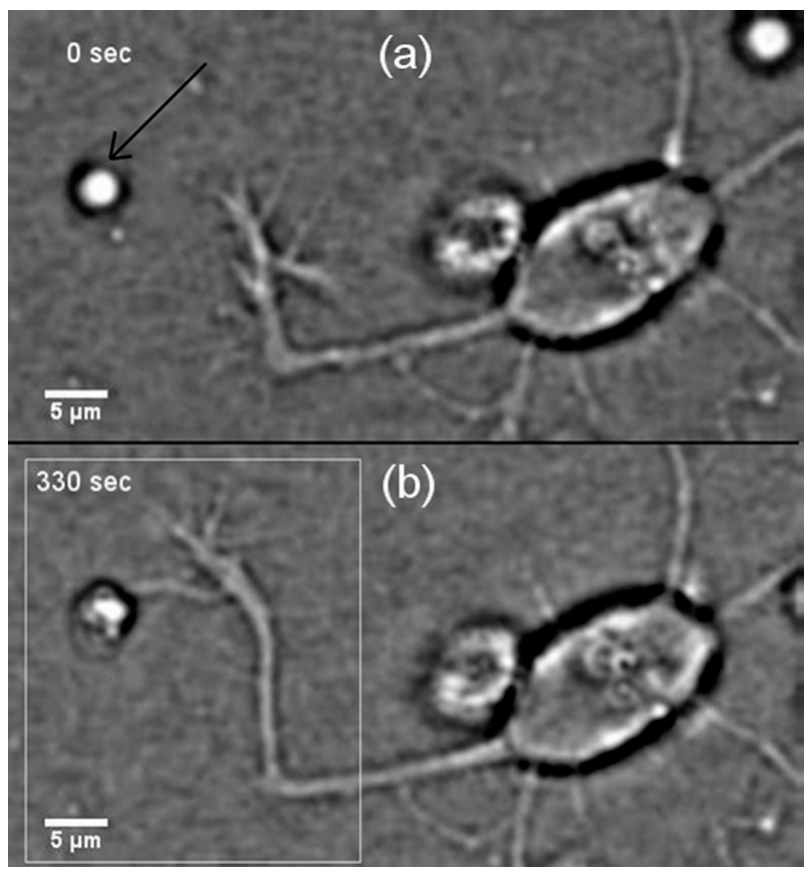

FIC. 3 Neurite guidance by Netrin-1 released from a micrometric size liposome (Movie-2, $162 \mathrm{~KB}$ ).

(a) A liposome (indicated by the black arrow) is positioned near a neurite of the neuron. The image was taken 30 seconds before liposome photolysis. Beside the neurite of interest, the soma and other neurites are visible.

(b) Neurite development 5 minutes after the release of the Netrin-1 molecules from the liposome. The progression of the region marked by the rectangle is shown in Figure 4 .

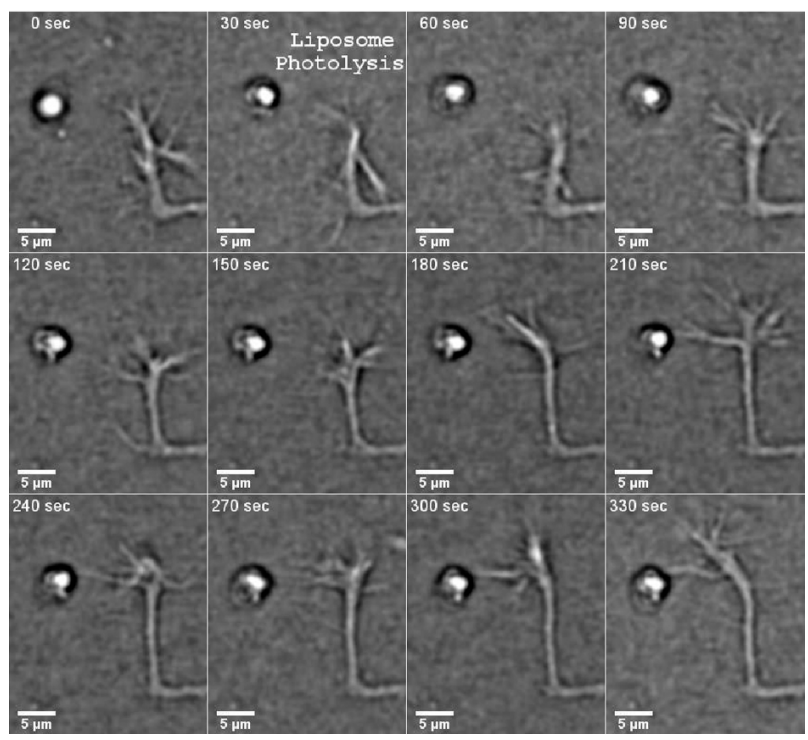

FIC. 4 Time lapse imaging of the local delivery of Netrin- 1 molecules encapsulated into a liposome and the corresponding chemotaxis effect induced on the axonal growth.

dynamically in Movie-2 (202 KB) and for a smaller rectangular region in Figure 4. A neat activation of the growth cone movement and the neurite progression toward the liposome location can be observed from this figure, demonstrating the chemotaxis effect. The growth of the neurite was about $10 \mu \mathrm{m}$ after 5 minutes.

We repeated the same experiment for the neurites of other five cells and observed effects similar to those of the neuron described above. The neurite movement was clearly ac- 
centuated after the release of Netrin-1 molecules and it was attracted toward the liposome location. In order to replicate the same experimental conditions we tried to select liposomes with the same diameter and position them at the same distance from the neurite. Another important aspect is the release of the molecules from the liposome. This depends on the liposome configuration and on the interaction between the liposome and the UV pulses. During the synthesis process, both liposomes with single and multiple layers can be obtained. If a complete release can be assumed for single layer liposomes, in the case of multiple layer liposomes we can not exclude that residual molecules still remain inside the liposome after photolysis. A quantitative evaluation of the molecules released should therefore be considered with care. For instance, in the experiment discussed here, a multi-layer liposome with a diameter of about $5 \mu \mathrm{m}$ was broken by interaction with UV laser pulses with the spot below $0.5 \mu \mathrm{m}$. Therefore, the liposome was probably not completely disintegrated, and residual Netrin-1 molecules remained trapped inside it. The molecules released by photolysis diffused in solution and arrived to the membrane receptors of the neurite, activating the transduction pathway underlying the guidance process. Unreleased molecules remain inactive inside the liposome, unless a new release induced by liposome photolysis.

\section{CONCLUSION}

We demonstrated the encapsulation of the physiological protein Netrin-1 in solution (concentration $5 \mu \mathrm{g} / \mathrm{mL}$ ) into micrometric liposomes. These liposomes were used as vectors, trapped and transported by optical tweezers to specific positions in the proximity of the neuron neurites. Netrin-1 molecules were released from the liposomes by photolysis with short UV laser pulses. The molecules released induced an accentuated movement of the neurite terminations and a development of the neurite toward the liposome site (chemotaxis effect).

The approach described in this paper presents several advantages which might be very useful in neurobiology. Since the volume of the liposomes can be tuned in a wide range, the number of molecules that can be encapsulated and transported by these vectors can be varied also in a wide range. This is of importance when trying to answer the question: whhat is the minimum amount of stimulating molecules which induces chemotaxis? If necessary, one can even think of a single molecule encapsulation and release. In fact, considering a concentration of $1 \mu \mathrm{g} / \mathrm{mL}$ and a liposome of radius $0.6 \mu \mathrm{m}$, which is feasible for optical manipulation, we get 1 molecule/liposome.

Another advantage of the technique is the positioning with sub-micrometric spatial resolution due to the use of optical tweezers. Time resolution is also very important in many experiments. The technique using the UV laser pulses, allows to define with high temporal resolution the initiation of the molecules release.

A final remark is that Netrin-1 molecules are not the only molecules possible to be encapsulated into liposomes. Indeed any molecule, with the exception of permeable membrane molecules, can be used, opening the way for a broad series of experiments in cell biology, in general, and neurobiology, in particular.

\section{References}

[1] M. M. Black, and P. W. Baas "The basis of polarity in neurons" Trends Neurosci. 12, 211 (1989).

[2] A. G. Pakhomov, I. Semenov, R. Brenner, and G. M. Toney “Hydraulically coupled microejection technique for precise local solution delivery in tissues" J Neurosci. Meth. 155, 231 (2006).

[3] S. Kim, H. J. Kim, and N. L. Jeon "Biological applications of microfluidic gradient devices" Integr. Biol. 2, 584 (2010).

[4] C. R. Kothapalli, E. van Veen, S. de Valence, S. Chung, I. K. Zervantonakis, F. B. Gertler, and R. D. Kamm "A high-throughput microfluidic assay to study neurite response to growth factor gradients" Lab Chip, Advance Article (2011).

[5] G. C. Ellis-Davies “Caged compounds: photorelease technology for control of cellular chemistry and physiology" Nat. Methods 4, 619 (2007).

[6] M. A. Smith, G. C. Ellis-Davies, and J. C. Magee "Mechanism of the distance-dependent scaling of Schaffer collateral synapses in rat CA1 pyramidal neurons" J. Physiol. 548, 245 (2003).

[7] M. Canepari, L. Nelson, G. Papageorgiou, J. E. Corrie, and D. Ogden "Photochemical and pharmacological evaluation of 7-nitroindolinyl-and 4-methoxy-7-nitroindolinyl-amino acids as novel, fast caged neurotransmitters" J Neurosci Meth. 112, 29 (2001).

[8] E. R. Dufresne, and D. G. Grier "Optical tweezer arrays and optical substrates created with diffractive optics" Rev. Sci. Instrum. 69, 1974 (1998).

[9] https://www.kisker-biotech.com/shop/microparticles-en.htm

[10] http://www.bangslabs.com/files/bangs/docs/pdf/205.pdf

[11] X.-h. Zhang, and M.-m. Poo "Localized synaptic potentiation by BDNF requires local protein synthesis in the developing axon" Neuron 36, 675, (2002).

[12] H. Kress, J. G. Park, C. 0. Mejean, J. D. Forster, J. Park, S. S. Walse, Y. Zhang, et al. "Cell stimulation with optically manipulated microsources" Nat. Methods 6, 905 (2009).

[13] B. Sun, and D. T. Chiu "Spatially and temporally resolved delivery of stimuli to single cells" J. Am. Chem. Soc. 125, 3702 (2003).

[14] P. Walde, and S. Ichikawa "Enzymes inside lipid vesicles: preparation, reactivity and applications" Biomol. Eng. 18, 143 (2001).

[15] J. L. Goldberg "How does an axon grow?" Gene. Dev.17, 941 (2003).

[16] V. Corset, K. T. Nguyen-Ba-Charvet, C. Forcet, E. Moyse, A. Chédotal, and P. Mehlen "Netrin-1 mediated axon outgrowth and CAMP production requires interaction with adenosine A2b receptor" $\mathrm{Na}$ ture 407,745 (2000).

[17] A. Steup, M. Lohrum, N. Hamscho, N. E. Savaskan, 0. Ninnemann, R. Nitsch, H. Fujisawa, et al. "Sema3C and netrin-1 differentially affect axon growth in the hippocampal formation" Mol. Cell. Neurosci. 15,141 (2000).

[18] K. C. Neuman, and S. M. Block "Optical Trapping - Review" Rev. Sci. Instrum. 75, 2787 (2004).

[19] V. Emiliani, D. Cojoc, E. Ferrari, V. Garbin, C. Durieux, M. CoppeyMoisan, and E. Di Fabrizio "Wave front engineering for microscopy 
of living cells" Opt. Express 13, 1395 (2005).

[20] D. Cojoc, V. Emiliani, E. Ferrari, R. Malureanu, S. Cabrini, and E. Di Fabrizio "Multiple optical trapping by means of diffractive optical elements" Jpn. J. Appl. Phys. 43, 3910 (2004).

[21] H. H. Hub, U. Zimmermann, and H. Ringsdorf “Preparation of large unilamellar vesicles" FEBS Lett. 140, 254 (1982).
[22] M. Ichikawa, and K. Yoshikawa "Optical transport of a single cellsized liposome" Appl. Phys. Lett. 79, 4598 (2001).

[23] M. E. Ruaro, P. Bonifazi, and V. Torre "Toward the neurocomputer: image processing and pattern recognition with neuronal cultures" IEEE Trans. Biomed. Eng. 52, 371 (2005). 